\title{
RESPOSTAS DA FREQUÊNCIA CARDÍACA DE JOVENS ATLETAS DE MODALIDADES COLETIVAS APÓS TESTES DE DESEMPENHO
}

\author{
Algicelia Sousa Ponte Guimarães ${ }^{1}$, Vitória Andrade Araújoº \\ Thainá Costa Barreto ${ }^{3}$, Lucas Guilherme Torres Ferreira ${ }^{4}$, \\ Ricardo Alexandre Rodrigues Santa Cruz
}

\begin{abstract}
Resumo: A frequência cardíaca (FC) é um indicador fisiológico amplamente utilizado para a avaliação, monitoramento e prescrição das intensidades dos esforços. Portanto, o objetivo do presente estudo foi analisar as respostas da FC de jovens atletas de modalidades coletivas em testes específicos de desempenho. Participaram do estudo 32 jovens atletas do sexo masculino $(16,4 \pm 0,35$ anos), praticantes de futsal que realizaram o Futsal Intermittent Endurance Test (FIET), voleibol que realizaram o TW20 metros Test e handebol que fizeram o Yo-yo recovery Test. $\mathrm{A} \mathrm{FC}_{\mathrm{PICO}}$ foi determinada como a mais alta FC encontrada ao final dos testes. Para testar a normalidade dos dados foram aplicados os testes de Shapiro Wilk e o teste $t$ de student para verificar a diferença entre a FC $_{\text {PICO }}$ nos diferentes testes de desempenho. Os resultados apontaram altas frequências cardíacas obtidas durante a realização dos testes por modalidade: futsal (FIET: 196,7* $\pm 5,89 \mathrm{bpm}$ ), voleibol (TW20: 187,8 \pm 9,69 bpm) e handebol (YO-YO: 190,5 \pm 8,68 bpm) sugerindo uma predominância do metabolismo anaeróbio. Concluímos que a $\mathrm{FC}_{\mathrm{PICO}}$ evidenciou valores máximos superiores para o futsal, e que a fórmula de Karvonen utilizada para predizer a FCmax superestima os valores reais quando empregadas com jovens atletas em testes de desempenho.
\end{abstract}

Palavras-chave: Frequência cardíaca. Testes de desempenho. Jovens atletas.

1 Graduanda em Educação Física pela Universidade Estadual de Roraima - UERR.

2 Graduanda em Educação Física pela Universidade Estadual de Roraima - UERR.

3 Graduando em Educação Física pela Universidade Estadual de Roraima - UERR.

4 Graduando em Educação Física pela Universidade Estadual de Roraima - UERR.

5 Doutor em Ciência do Movimento Humano; Professor do Curso de Educação Física pela Universidade Estadual de Roraima - UERR. 


\section{INTRODUÇÃO}

A frequência cardíaca (FC) é um indicador fisiológico amplamente utilizado para a avaliação, monitoramento e prescrição das intensidades dos esforços (CAMARDA et al., 2008). Por sua facilidade de mensuração, a FC tem sido uma das variáveis internas mais frequentemente empregadas para caracterizar a obtenção de um esforço máximo (ARAÚJO; PINTO, 2005).

De acordo com Wilmore e Costill (2013) a frequência cardíaca máxima $\left(\mathrm{FC}_{\mathrm{MAX}}\right)$ é o valor mais elevado da frequência cardíaca que um indivíduo pode atingir em um esforço máximo até o ponto de exaustão. $\mathrm{A} \mathrm{FC}_{\mathrm{MAX}}$ é uma das variáveis fisiológicas mais utilizadas para prescrição de cargas de trabalho em programas de exercícios, por demonstrar estreita relação com o consumo máximo de oxigênio (CAPUTO; SILVA; ROMBALDI, 2012).

Existem várias equações que predizem a $\mathrm{FC}_{\mathrm{MAX}}$ entretanto as mais conhecidas e utilizadas são as equações de Karvonen $\left(\mathrm{FC}_{\mathrm{MAX}}=220\right.$-idade $)$ e Tanaka $\left(\mathrm{FC}_{\mathrm{MAX}}=208-(0,7 \times\right.$ idade $\left.)\right)$, embora se encontrem divergências na literatura sobre sua aplicabilidade, com relação às estimativas, podendo subestimar ou superestimar o escore da $\mathrm{FC}_{\mathrm{MAX}}$ em relação ao valor obtido diretamente.

Gonçalves e Santana (2013) apontam que a avaliação da demanda metabólica em modalidades esportivas intermitentes, torna-se necessária para a compreensão de como o desempenho e o treinamento devem ser realizados. Nesse sentido, a avaliação da capacidade cardiorrespiratória nos esportes é uma prática muito comum para estabelecer níveis de condicionamento e poder melhorá-los (ZANELLA et al., 2015).

Avaliar e monitorar precisamente a intensidade imposta aos atletas em testes de campo ao longo da temporada é um importante aspecto para a efetividade da periodização e para a prevenção de efeitos negativos, como a queda no rendimento.

Os testes de campo com características próprias da modalidade foram criados como forma de avaliação e também prescrição de treinamento e são recomendados pela especificidade e validade ecológica que possuem (ARINS et al., 2015). Bergamasco et al. (2005), ressaltam que a busca por avaliações que possam refletir a condição física dos atletas em determinada modalidade vem sendo pesquisada há algum tempo, e apontam ainda que vários são os índices que podem ser mensurados para expressar essa condição física, dentre eles a FC. Portanto, o objetivo do presente estudo foi analisar as respostas da FC de jovens atletas de modalidades coletivas em testes específicos de desempenho. 


\section{MÉTODOS}

\section{Participantes}

A amostra participante do estudo foi composta por 32 jovens atletas do sexo masculino (16,4 $\pm 0,35$ anos), praticantes de modalidades coletivas de quadra (Futsal $=10 ;$ Voleibol $=14 ;$ Handebol $=08$ ). Os atletas apresentavam uma frequência de treinamentos de três a quatro vezes por semana, com volume total de aproximadamente oito horas semanais de atividades específicas da sua modalidade e disputavam competições estaduais.

De acordo com a resolução 466/12 do Conselho Nacional de Saúde o estudo foi aprovado pelo Comitê de Ética em Pesquisa da Universidade Estadual de Roraima - CEP-UERR, sob parecer número 1.801.214/2016.

Anteriormente à aplicação dos testes de desempenho, as comissões técnicas das equipes, os atletas e seus responsáveis legais foram esclarecidos em relação aos objetivos e procedimentos do estudo, assinando os termos de assentimento e consentimento livre e esclarecido respectivamente.

\section{Avaliações antropométricas}

As avaliações antropométricas foram realizadas antes da aplicação dos testes de desempenho, no próprio local de treinamento das equipes. A massa corporal foi mensurada utilizando-se uma balança eletrônica, com precisão de $0,1 \mathrm{~kg}$, e a estatura foi determinada em um estadiômetro portátil, com precisão de $0,1 \mathrm{~cm}$. Para o cálculo do IMC foi utilizado a seguinte fórmula: peso/altura ${ }^{2}$ $\left(\mathrm{kg} / \mathrm{m}^{2}\right)$.

\section{Desenho Experimental do Estudo}

Para analisar as respostas da FC dos atletas, foram utilizados testes de campo, observando a especificidade e a caracterização dos gestos motores da modalidade na execução dos testes. Dessa forma, os atletas de futsal realizaram o Futsal Intermittent Endurance Test (FIET), os atletas de voleibol realizaram o TW20 metros Test e os atletas de handebol o Yo-yo recovery Test Nível I.

As avaliações antropométricas e os testes de desempenho foram realizados sob a supervisão da comissão técnica, na quadra de treinamentos das equipes.

\section{Protocolo do Futsal Intermittent Endurance Test (FIET)}

O FIET foi proposto por Álvarez e Ándrin (2005) e validado por Castagna e Álvarez (2010). O teste foi realizado de acordo com os procedimentos descritos por Carminatti et al. (2015), consiste de repetições de corridas em sistema de shuttle run de 45 metros ( 3 × 15 metros), as quais são intercaladas 
por 10 segundos de recuperação, sendo que há um período de pausa maior (30 segundos) após cada estágio. A velocidade inicial do teste é de $9 \mathrm{~km} \mathrm{~h}$, com incrementos de $0,33 \mathrm{kmh}$ durante as nove primeiras repetições $(9 \times 45$ metros), mudando na sequência para $0,20 \mathrm{kmh}$ a partir da décima repetição de 45 metros. O ritmo é ditado por um sinal sonoro (bip), que determina a velocidade de corrida a ser desenvolvida nos deslocamentos entre as linhas paralelas demarcadas no solo (15 metros) e também sinalizadas por cones. $\mathrm{O}$ teste é encerrado quando o avaliado atrasar mais do que 1,5 metros em relação à linha de referência de 15 metros por duas vezes consecutivas, ou então, no momento que o mesmo atingir a exaustão voluntária.

\section{Protocolo do TW20 metros Test}

O teste TW20 metros foi proposto por Pellegrinotti e Souza (2001). O teste tem duração fixa de 6 minutos sendo realizado na quadra de voleibol, na zona de ataque dos três metros, com a rede na altura estabelecida para a categoria masculina. No teste, o atleta realiza uma corrida de 18,80 metros correndo de frente, com percurso composto por três bloqueios de $40 \mathrm{~cm}$ (na entrada, meio e saída de rede), sendo $20 \mathrm{~cm}$ para subir e $20 \mathrm{~cm}$ para descer, realizados por pontos demarcados entre a linha dos três metros e a rede, perfazendo cinco mudanças de direção, completando assim um estágio em forma de "W". Ao final do tempo de teste é registrado: a máxima distância alcançada pela passagem no percurso ininterruptamente, em metros; a quantidade de saltos e o número de mudanças de direção.

\section{Protocolo do Yo-yo recovery Test}

O Yo-yo recovery test nível I foi proposto por Bangsbo (1996). O teste consiste em percorrer a distância de 20 metros em regime de ida e volta $(40 \mathrm{~m})$ com um intervalo de $10 \mathrm{~s}$ de recuperação ativa, em $5 \mathrm{~m}$. A velocidade inicial do teste é de $10 \mathrm{~km} \bullet \mathrm{h}^{-1}$, a segunda velocidade é de $11,5 \mathrm{~km} \bullet \mathrm{h}-{ }^{1}$ e nas velocidades seguintes, a partir de $13 \mathrm{~km} \bullet \mathrm{h}^{-1}$, a velocidade aumenta em $0,5 \mathrm{Km} \bullet \mathrm{h}^{-1}$, até a exaustão. A velocidade do teste foi controlada por sinais sonoros emitidos por mídia. $\mathrm{O}$ teste foi interrompido quando os jogadores não foram capazes de percorrer por duas vezes consecutivas o trajeto dentro do tempo delimitado pelos sinais sonoros ou pela fadiga voluntária, sendo considerada a distância percorrida total (em metros) e a maior velocidade atingida como desempenho final do teste.

\section{Monitoramento da Frequência Cardíaca}

Durante a realização dos testes de desempenho, os atletas utilizaram monitores de frequência cardíaca (GARMIN, Vivofit@). As pulseiras transmissoras foram colocadas no punho e os cardiofrequencímetros foram 
colocados junto ao peito dos atletas antes do início dos testes. Ao término da avaliação, eles eram recolhidos pelo pesquisador.

A FC atingida pelos atletas ao final dos testes de desempenho foi considerada como $\mathrm{FC}_{\mathrm{PICO}}$. Para efeito de comparação da intensidade alcançada ao final da execução dos testes de esforço pelos atletas, calculou-se indiretamente as frequências cardíacas máximas pelas equações de predição de Karvonen $\left(\mathrm{FC}_{\mathrm{MAX}}=220\right.$ - idade $)$ e Tanaka $\left(\mathrm{FC}_{\mathrm{MAX}}=208-(0,7 \times\right.$ idade $)$ ).

\section{Análise dos dados}

Foram determinadas as médias e desvios padrão das variáveis idade, massa corporal, estatura, índice de massa corpórea e $\mathrm{FC}_{\mathrm{PICO}}$ atingida nos testes de desempenho. Para testar a normalidade dos dados foram aplicados os testes de Shapiro Wilk e o teste $t$ de student para verificar a diferença entre a $\mathrm{FC}_{\mathrm{PICO}}$ nos diferentes testes de desempenho. Os dados foram analisados estatisticamente no software SPSS. Foi adotado um nível de significância de $\mathrm{p}<0,05$.

\section{RESULTADOS}

Para caracterizar a amostra, foi identificada a idade dos atletas pelo ano de nascimento e realizadas as medidas do peso da massa corporal $(\mathrm{Kg})$ estatura $(\mathrm{cm})$ e calculou-se o índice de massa corpórea $\left(\mathrm{kg} / \mathrm{m}^{2}\right)$. Os valores dessas variáveis são apresentados em médias e desvio padrão na tabela 1.

Tabela 1 - Características gerais dos atletas de Futsal, Voleibol e Handebol

\begin{tabular}{l|c|c|c}
\hline Variáveis & Futsal & Voleibol & Handebol \\
\hline Idade (anos) & $16,8 \pm 0,89$ & $16,1 \pm 0,64$ & $16,3 \pm 0,38$ \\
Massa Corporal $(\mathrm{kg})$ & $62,1 \pm 5,58^{*}$ & $67,4 \pm 8,79$ & $67,1 \pm 6,35$ \\
Estatura $(\mathrm{cm})$ & $166 \pm 0,05$ & $177 \pm 0,05^{*}$ & $172 \pm 4,58$ \\
IMC $\left(\mathrm{kg} / \mathrm{m}^{2}\right)$ & $22,1 \pm 1,98$ & $21,3 \pm 0,92$ & $22,6 \pm 0,93$ \\
\hline
\end{tabular}

* Diferença significante para as outras modalidades $(P>0,05)$

A tabela 2, apresenta os valores comparativos da $\mathrm{FC}_{\mathrm{PICO}}$ obtida nos testes específicos de desempenho, $\mathrm{FC}_{\mathrm{MAX}}$ estimada pela fórmula de Karvonen (220-idade), $\mathrm{FC}_{\mathrm{MAX}}$ estimada pela fórmula de Tanaka (208-0,7x idade) entre os atletas de futsal, voleibol e handebol 
Tabela 2 - Comparação entre os valores da FCpico ao final dos testes de esforço, e FC estimada pelas fórmulas de KARVONEN e TANAKA

\begin{tabular}{l|c|c|c}
\hline Variáveis & $\begin{array}{c}\text { Futsal } \\
\text { FC }_{\text {MÁx FIET }}\end{array}$ & $\begin{array}{c}\text { Voleibol } \\
\text { FC }_{\text {MÁx TW 20 }}\end{array}$ & $\begin{array}{c}\text { Handebol } \\
\text { FC }_{\text {MÁx YO-YO }}\end{array}$ \\
\hline FCpico (bpm) & $196,7 \pm 5,89^{*}$ & $187,8 \pm 9,69$ & $190,5 \pm 8,68$ \\
FC $_{\text {KARVONEN }}$ & $203,2 \pm 2,53$ & $203,9 \pm 3,02$ & $203,7 \pm 4,35$ \\
FC $_{\text {TANAKA }}$ & $196,2 \pm 3,42$ & $196,7 \pm 4,89$ & $196,5 \pm 3,55$ \\
\hline
\end{tabular}

Legenda: $\mathrm{FC}_{\mathrm{MAXFIET}}=$ Frequência cardíaca máxima alcançada no Futsal Intermittent Endurance Test; $\mathrm{FC}_{\mathrm{MAXTW20}}=$ Frequência cardíaca máxima alcançada no TW20Test; $\mathrm{FC}_{\mathrm{MAXYO}-\mathrm{YO}}=$ Frequência cardíaca máxima alcançada no Yo-yo recovery Test nível I; *Diferença significante para as outras modalidades $(\mathrm{P}>0,05)$.

\section{DISCUSSÃO}

O objetivo principal desse estudo foi analisar as respostas da FC de jovens atletas de modalidades coletivas em testes específicos de desempenho. Considerando esse objetivo, buscou-se avaliar e descrever os valores da $\mathrm{FC}_{\text {PICO }}$ obtidos em testes de desempenho para as modalidades de futsal, voleibol e handebol, na tentativa de mensurar a carga de trabalho fisiológico imposto durante os testes.

Ao analisarmos a tabela 2, verifica-se que a $\mathrm{FC}_{\mathrm{MAX}}$ predita pela equação de Karvonen foi significativamente maior $(p<0,001)$ que a encontrada com a utilização da equação de Tanaka e pelos testes de desempenho.

Caputo, Silva e Rombaldi (2012) ressaltam que a fórmula "FCmax = 220-idade" de Karvonen tem certa tendência a superestimar a FCmax em indivíduos jovens e a subestimá-la em indivíduos idosos. Essa afirmativa é confirmada no presente estudo.

Nos testes de desempenho, os resultados apontaram altas frequências cardíacas obtidas durante a realização dos testes por modalidade: futsal (FIET: 196,7 \pm 5,89 bpm), voleibol (TW20: 187,8 \pm 9,69 bpm) e handebol (YO-YO: 190,5 $\pm 8,68 \mathrm{bpm}$ ) sugerindo uma predominância do metabolismo anaeróbio ao final dos testes.

Por meio do teste $\mathrm{t}$ Student, para dados pareados, foi encontrada diferença significativa $(\mathrm{p}<0,05)$ para a $\mathrm{FC}_{\text {PICO }}$ obtida no FIET para os atletas de futsal, quando comparada com os atletas de voleibol e handebol. A FC dos atletas de futsal ao final do FIET foi muito aproximada com a estabelecida pela equação de Tanaka (196,2 $\pm 3,42 \mathrm{bpm})$, indicando uma possibilidade de se encontrar a $\mathrm{FC}_{\mathrm{MAX}}$ de jovens atletas de futsal pelo FIET. 
Utilizando o FIET para avaliar a intensidade dos esforços no futsal feminino, Carminatti et al. (2015) relataram índices de $\mathrm{FC}_{\text {MAXFIET }}$ de $196 \pm 9,00$ bpm, valores esses aproximados dos obtidos no monitoramento da $\mathrm{FC}_{\mathrm{MAX}} \mathrm{em}$ jogos oficiais e simulados (197 $\pm 8,00 \mathrm{bpm})$.

Estes resultados são parecidos aos encontrados no presente estudo $(196,7 \pm$ $5,89 \mathrm{bpm}$ ) com jovens atletas juvenis de futsal avaliados com o FIET, indicando que avaliar as capacidades aeróbia/anaeróbia de atletas de futsal por meio desse teste, revela intensidades de $\mathrm{FC}_{\mathrm{MAX}}$ semelhantes as encontradas em partidas oficiais.

Já Gonçalves e Santana (2013) encontraram valores inferiores de $\mathrm{FC}_{\mathrm{MAX}}$ em atletas de elite brasileiros durante jogos oficiais de futsal $(181,00 \pm 7,57$ bpm). Resultados semelhantes, foram descritos por Nunes et al. (2012) ao avaliarem atletas profissionais de futsal da categoria adulta em teste de esteira incremental, encontrando resultados da $\mathrm{FC}_{\mathrm{MAX}}$ no último estágio completo do teste de 183,1 $\pm 8,7 \mathrm{bpm}$, indicando dessa forma, boa correlação entre testes de campo e laboratório para as respostas da $\mathrm{FC}_{\mathrm{MAX}}$, porém com menores percentuais quando comparados com o futsal feminino e com jovens atletas.

No voleibol, utilizando o teste TW20 metros, as respostas da $\mathrm{FC}_{\mathrm{PICO}}$ encontradas no presente estudo apontaram valores de 187,8 \pm 9,69 bpm, como média ao final da realização do teste. Esses achados são parecidos aos encontrados por Nascimento et al., (2013) com atletas paulistas de voleibol feminino de elite $(182,6 \pm 6,04 \mathrm{bpm})$. Os autores enfatizam que o teste TW20 metros implica em uma alta contribuição do sistema glicolítico, e a mensuração da $\mathrm{FC}_{\mathrm{MAX}}$ ao final da avaliação pode predizer a intensidade dos esforços que são encontrados em jogos de voleibol.

Quando os resultados da $\mathrm{FC}_{\text {PICO }}$ obtidos no TW20 são comparados com as fórmulas de Karvonen (203,9 $\pm 3,02 \mathrm{bpm})$ e Tanaka $(196,7 \pm 4,89 \mathrm{bpm})$ os valores são superestimados.

No handebol, analisando as respostas da $\mathrm{FC}_{\text {PICO }}$ dos jovens atletas submetidos ao Yo-yo recovery Test nível I, foram encontrados valores de 190,5 $\pm 8,68 \mathrm{bpm}$ ao final do teste. Quando esse valor é comparado com a fórmula de Karvonen, pode-se identificar uma subestimação da $\mathrm{FC}_{\mathrm{MAX}}$ dos atletas $(203,7$ $\pm 4,35 \mathrm{bpm}$ ) e uma aproximação aos valores referenciados na estimativa de Tanaka (196,5 $\pm 3,55 \mathrm{bpm})$.

Bergamasco et al. (2005) avaliou a $\mathrm{FC}_{\mathrm{MAX}}$ de universitários do sexo masculino atletas de handebol ao final do teste progressivo de vai e vem (LĖGER; LAMBERT, 1992) e comparou os resultados com a equação de predição de Karvonen. Os resultados apontaram valores de 200,3 \pm 5,1 bpm ao final do teste, superiores aos encontrados no presente estudo com atletas da categoria juvenil. Quando a comparação foi realizada pela fórmula de Karvonen encontrou-se valores de 197,8 $\pm 3,55$ bpm. 
Os autores afirmam que para a predição da $\mathrm{FC}_{\mathrm{MAX}}$ em indivíduos treinados torna-se necessário a utilização de testes com maior precisão para estimativa.

\section{CONCLUSÃO}

De acordo com os resultados, foi possível concluir, pela comparação entre as médias dos valores de $\mathrm{FC}_{\text {PICO }}$ entre os atletas das modalidades coletivas analisadas, que os jogadores de futsal submetidos ao FIET obtiveram valores superiores de $\mathrm{FC}_{\mathrm{MAX}}$ quando comparados com os atletas de voleibol pelo teste TW20 metros e pelos atletas de handebol pelo Yo-yo recovery Test nível I. Os resultados ainda indicam que a fórmula de Karvonen utilizada para predizer a $\mathrm{FC}_{\mathrm{MAX}}$ superestima os valores reais quando empregadas com jovens atletas em testes de desempenho.

\section{REFERÊNCIAS}

ÁLVAREZ, J.C.B.; ANDRÍN, G. Desarrollo y aplicación de un nuevo test de campo para valorar la resistencia específica en jugadores de fútbol sala: TREIF (Test de Resistencia específica intermitente para Fustal). Lecturas, Educación Física y Deportes, 2005.

ARAÚJO, C.G.S.; PINTO, V.L.M. Frequência Cardíaca Máxima em Testes de Exercício em Esteira Rolante e em Cicloergômetro de Membros Inferiores. Arquivos Brasileiros de Cardiologia, v.85, n.1, p. 45-50, 2005.

ARINS, F.B. Physiological characteristics, evaluation and prescription of aerobic training in Futsal. Revista Brasileira de Cineantropometria \& Desempenho Humano, v.17, n.6, p.753-762, 2015.

BANGSBO, J. Yo-Yo tests. Copenhagen: August Krogh Institute, 1996.

BERGAMASCO, J. G. P. et al. Análise da frequência cardíaca e do VO2 máximo em atletas universitários de handebol através do teste do Vai-e-Vem 20 metros. Movimento \& Percepção, Espírito Santo do Pinhal, v.5, n. 7, p. 146-163, 2005.

CAMARDA, S.R.A. Comparação da frequência cardíaca máxima medida com as fórmulas de predição propostas por Karvonen e Tanaka. Arquivos Brasileiros em Cardiologia, v. 91, n. 5, p. 311-314, 2008.

CAPUTO, E.L.; SILVA, M.C.; ROMBALDI, A.J. Comparação da frequência cardíaca máxima obtida por diferentes métodos. Revista Educação Física UEM, v.23, n.2, p.277-284, 2012.

CARMINATTI, L.J. et al. Intensidade de esforço em jogos oficiais e simulados de futsal feminino. Revista Brasileira de Ciência e Movimento, v.23, n.3, p.97-104, 2015. 
CASTAGNA, C.; ÁLVAREZ, J.C.B. Physiological Demands of an Intermittent FutsalOriented High-Intensity Test. Journal of Strength and Conditioning Research, n.0, v.0, p.1-8, 2010.

GONÇALVES, H.R.; SANTANA, W.C. Valores de frequência cardíaca de jogadores de futsal em situação de jogo. Pensar a Prática, v. 16, n.1, p.13-19, 2013.

LÈGER, L.A.; LAMBERT, J. A maximal multistage 20 - m shuttle run test to predict VO2 máx. European Journal Physiological, n. 49, p. 1-12, 1982.

NASCIMENTO T.A. et al. Evaluation of physical capacity in athletic female volleyball players using the TW20meters test. Gazzetta Medica Italiana. v.172: p. 449-455, 2013.

NUNES, R.F. et al. Comparação de indicadores físicos e fisiológicos entre atletas profissionais de futsal e futebol. Motriz, v.18, n.1, p.104-112, 2012.

PELLIGRINOTTI, I.L.; SOUZA, S.J. Criação do "teste w 20 metros" e instrumento computadorizado para avaliação da performance de voleibolistas. Revista Treinamento Desportivo, v.5, n.1, p. 16-28, 2001.

TANAKA, H.; MONAHAN, D.K.; SEALS, R.D. Age Predicted Maximal Heart Rate Reviset. Journal College Cardiology, v. 37, n. 1, p. 153-156, 2001.

WILMORE, J.H, COSTILL, D.L. Fisiologia do esporte e do exercício. $5^{\text {a }}$ ed. São Paulo: Manole; 2013.

ZANELLA, A.L. et al. Consumo máximo de oxigênio de jogadores de voleibol: comparação entre posições de jogo. Revista Brasileira de Prescrição e Fisiologia do Exercício, v. 9. n. 54. p. 404-409, 2015. 